\title{
Measurement-Based Admission Control in Wireless Sensor Networks
}

\author{
Ibrahim Orhan \\ School of Technology and Health \\ $\mathrm{KTH}$ \\ Stockholm, Sweden \\ Ibrahim.Orhan@sth.kth.se
}

\author{
Thomas Lindh \\ School of Technology and Health \\ $\mathrm{KTH}$ \\ Stockholm, Sweden \\ Thomas.Lindh@sth.kth.se
}

\begin{abstract}
Wireless sensor networks have today emerged as a feasible infrastructure for healthcare applications. This paper addresses the non-trivial performance problems in contentionbased wireless networks. We present a method for admission control in contention-based networks, implemented as a component of a performance management system. The test results show that admission control can improve the predictability and level of performance in wireless sensor networks. The system can be used as a tool for dimensioning and configuration as well as for real-time admission control. The often unpredictable dynamics in contention-based access networks means that continuous performance control is needed to maintain a desired quality of service.
\end{abstract}

Keywords-wireless sensor networks, admission control, performance monitoring and control.

\section{INTRODUCTION}

Wireless personal area networks have emerged as an important communication infrastructure in areas such as athome healthcare and home automation, independent living and assistive technology, as well as sports and wellness. Initiatives towards interoperability and standardization are taken by several players e.g., in healthcare applications. Zigbee Alliance has launched a profile for "Zigbee wireless sensor applications for health, wellness and fitness" [1]. The Continua Health Alliance promotes "an interoperable personal healthcare ecosystem" [2], and at-home health monitoring is also discussed in an informational Internet draft [3]. It shows that wireless personal area networks, including body sensor networks, are becoming more mature and are considered to be a realistic alternative as communication infrastructure for demanding services. However, to transmit data from e.g., an ECG in wireless networks is also a challenge, especially if multiple sensors compete for access as in CSMA/CA. Contention-based systems offer simplicity and utilization advantages, but the drawback is lack of predictable performance. This paper discusses whether admission control in combination with a system for continuous performance management can provide improved and more predictable performance.

Admission control is used in many traditional telecom systems. It is also proposed in new Internet service architectures [4] to provide guarantees for quality of service. In this paper we present a method for measurement-based admission control in wireless personal area sensor networks for contention-based access. It is implemented as a part of an integrated performance management system that also com- prises performance monitoring, admission control and performance control.

The rest of the paper is organized as follows: a survey of related work in Section II; performance management in wireless sensor networks in Section III; measurement-based admission control in Section IV; use cases and test results in Section V; and finally the conclusions in Section VI.

\section{RELATED WORK}

Our approach is to base the decision, to accept or reject a request to join the network, on measurements of performance parameters, mainly the packet loss ratio. A similar probebased admission control procedure has been suggested for differentiated internet services [4]. Alternatively, one can measure the available capacity between two endpoints or on certain links in a network. Pathrate, Pathload and BART are examples of implementations of such estimation tools ([5], [6] and [7]). SenProbe [8] estimates the maximum achievable rate between two endpoints in wireless sensor networks by injecting packet trains and analysing the dispersion between the packets. Some experimental studies indicate that measurements of available capacity in wireless networks often give inaccurate results especially for multiple hops [9]. Instead of active measurements, Contention-aware admission control protocol (CACP) estimates the available capacity by measurements from each node of the amount of time the channel is busy [10]. Perceptive admission control (PAC) is an extension of CACP to encompass node mobility [11]. We have preferred a straightforward approach where the decision to either accept or reject an admission request is based on direct measurements and estimates of the performance parameters that are decisive for the quality of services.

\section{PERFORMANCE MANAGEMENT IN WIRELESS SENSOR NETWORKS}

Admission control needs to be seen in the context of other necessary functions, especially performance measurements and control. In this section we briefly present the performance management system that admission control is a part of. The performance manager consists of the following functions: a performance meter that collects measurement data; admission control that handles requests to join the network; and performance control that maintains the quality of service for the admitted sensor nodes. The performance meter provides feedback measurement data for admission control and performance control. Fig. 1 shows the relation- 
ship between these functions. A request from a sensor node to join the network is handled by the admission control based on feedback from the meter. The performance control function is responsible for maintaining the desired quality of service once the sensors are allowed to use the wireless channel.

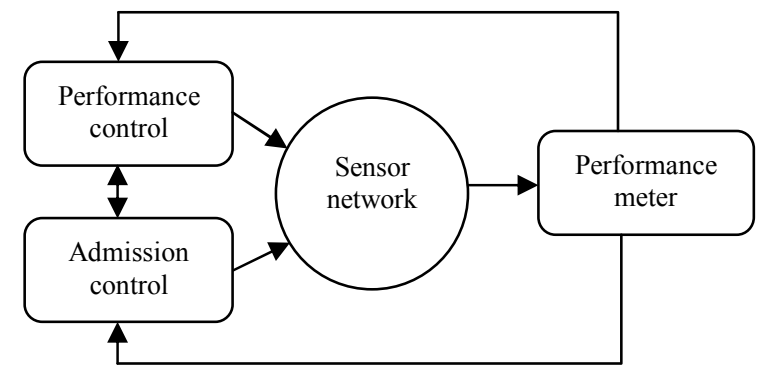

Figure 1. The performance manager consists of admission control and performance control. The performance meter supports the manager with measurement data.

\section{A. The Performance Meter}

The performance meter, which is based on so called monitoring blocks (Fig. 2), is implemented in the sensor nodes and the coordinator (Fig. 3). It consists of counters that keep track of the number of sent and received packets and bytes, and a function that can insert monitoring packets. These monitoring packets are inserted between blocks of ordinary data packets as seen in Fig. 2. They contain a sequence number, a timestamp and the cumulative number of packets and bytes transmitted from the sending node to the receiving node. The interval between the monitoring packets i.e., the size of the monitoring block, can be expressed in number of packets or a time interval. When a monitoring packet arrives, the receiving coordinator will store a timestamp and the cumulative number of received packets and bytes from the sending node. The following metrics can be calculated and estimated based on the collected measurements: packet loss, inter-arrival jitter and throughput. Detailed information and test results are presented in [12].

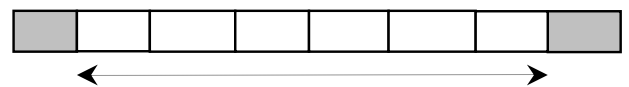

Figure 2. A monitoring block containing data packets surrounded by two monitoring packets.

\section{B. Feedback Performance Control}

The performance manager, implemented in the coordinator, uses the measurement feedback it receives from the meter: packet loss, delays and throughput. The meter delivers these performance updates for each incoming monitoring block e.g., once a second. The performance manager can order sensor nodes to decrease their transmission to maintain the quality of service for the connected nodes. Sensor nodes can also be assigned different priority. If a high-priority sensor experiences losses above a specified threshold, the manager can instruct low-priority sensors to back-off and decrease their sending rate. The control algorithm has three main tuneable parameters that determine the responsiveness of the control actions: the size of the monitoring blocks; the number of previous monitoring blocks used by the algorithm; and the step size to decrease or increase the packet frequency. More details on the performance manager are presented in [13].

\section{ADMISSION CONTROL IN WIRELESS SENSOR NETWORKS}

In this section the main idea behind the admission control system is presented.

\section{A. An Application Scenario}

A typical application scenario is healthcare at-home with a number of sensors such as ECGs, pulse-oximeters, accelerometers etc connected to a coordinator. Fig. 3 shows a case with three sensors connected to a coordinator sharing the same wireless channel that applies the CSMA/CA access method. Several hops between the sensors and the coordinator including mobility are also a feasible. Sensor A and sensor B in Fig. 3 are already connected to the wireless channel transmitting sensor data to the coordinator. The sensors have a priority levels, a specified throughput and an upper limit for the packet loss ratio. Sensor $\mathrm{C}$ accesses the channel and requests admission for a specified throughput and related packet loss requirements.

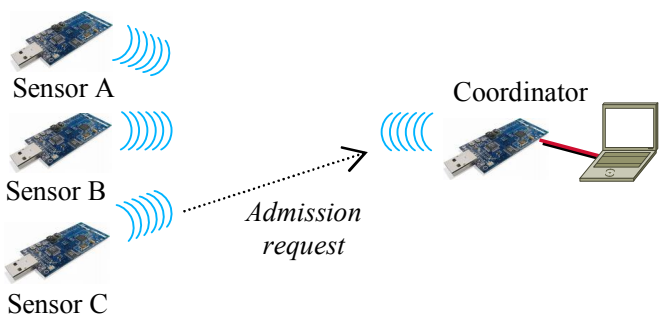

Figure 3. Sensor $\mathrm{C}$ requests to share the wireless channel already used by sensor $\mathrm{A}$ and sensor $\mathrm{B}$.

\section{B. Measurement-Based Admission Control for Contention- Based Access}

The purpose of admission control is to accept or reject new sensor nodes to an existing network, while protecting the performance of already admitted nodes. This paper focuses on whether it is feasible or not to use admission control in contention-based wireless sensor networks. Our approach is to found the decision to accept or reject an admission request on estimates of real-time measurement data provided by a performance meter. A sensor node that intends to enter the network specifies the sampling rate, the sample size and the performance requirements. The verdict, to accept or reject the request, is determined by the outcome of probe packets transmitted during a test period. The test traffic sent from the requesting node to the coordinator should be the same as the ordinary traffic it will transmit if admitted. The exchanged messages between a requesting node and the coordinator are described in the next subsection. 
In contention-based access networks, such as CSMA/CA for IEEE 802.15.4, strict performance guarantees are not feasible. However, many applications do not require completely loss-free transmission and are satisfied with soft performance requirements e.g., upper limits on packet loss and delay variation. This paper addresses the need for performance guarantees and predictability in contention-based networks for such applications.

1) Messages between the coordinator and sensor nodes

A simple protocol for exchange of messages between the coordinator and the sensor nodes has been defined (see Fig. 4). Sensor nodes send requests to join the network for a specified sampling rate, sample size, priority and upper limits on performance parameters. If the coordinator is not busy handling previous requests, it will approve further processing. The sensor node is then instructed to start transmitting test traffic, including monitoring packets. When the test period is ended, the sensor node asks the coordinator for the decision. Having received 'accept', the sensor node begins transmitting its ordinary data packets to the coordinator. Monitoring packets are inserted between blocks of $n$ data packet or with a certain time interval, to provide the performance meter at the coordinator with real-time updates of the transmission quality.

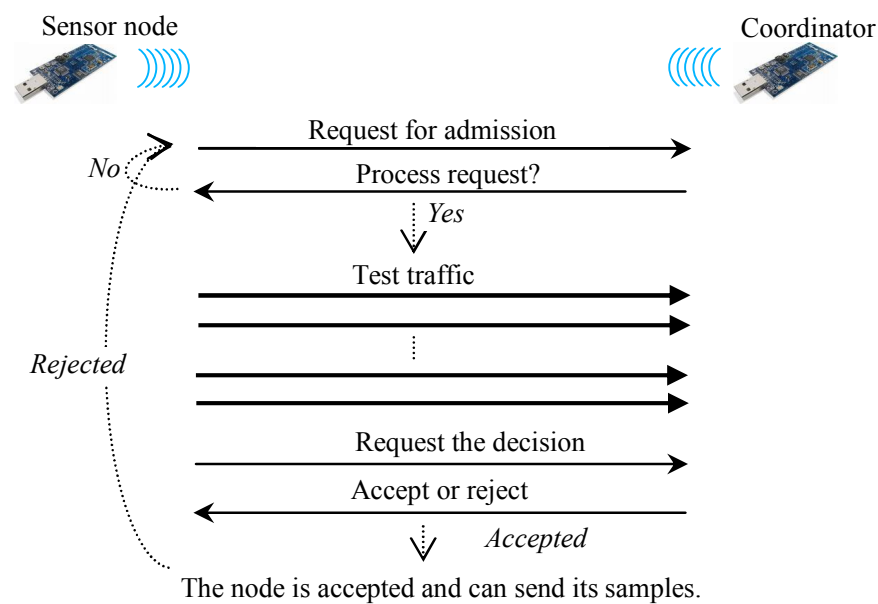

Figure 4. The messages between a sensor node and the coordinator during the admission phase. The arrows in thin lines are signalling messages and the arrows in thick lines represent test traffic.

\section{2) Admission test period}

The sensor nodes transmit packets during the test period in the same way as they intends to do if the request is accepted e.g., with a $200 \mathrm{~Hz}$ sampling rate and 12 bits sample size. The performance meter will report the performance data for traffic between the coordinator and all sensor nodes, including the test traffic from the requesting sensor. Admission is accepted if the average of the performance parameters for any of the already permitted nodes, as well as for the requesting node, is below the threshold value. Admission can be denied to protect the existing nodes from performance degradation.
The length of the test period is a trade-off between retrieving enough information from the probe packets and minimizing the effect on the other sensor nodes' performance. The first priority is to protect the already admitted nodes. The test traffic will be interrupted as soon as the disturbance of the probe packets exceeds the specified thresholds.

The probe packets sent during the test period represent a sampling process of the wireless channel, where the outcome of each sample event is that the packet is lost or not. The probability to lose a packet depends on the total traffic load and the number of nodes transmitting (ignoring radio channel disturbances). The number of samples needed for a given confidence level is determined by the variation of the traffic load. We have assumed that the sampling frequencies of the sensors are stable. This is a reasonable assumption for the kind of the applications the system is intended for. It means that the variance of the traffic load over time is low, and accordingly, that the number of probe packets can be kept low. The experiences from the test cases (Section V) in a normal home environment confirm that a test period of less than 30 seconds is sufficient. The length of the test period is further discussed in Section V.B.

\section{3) Applications}

The method and system described in this paper can be used for admission control and continuous real-time performance monitoring and control of operating wireless sensor networks. In addition, it is suitable for dimensioning, configuration and testing of wireless sensor networks prior to operational mode. The system can determine the number of sensors with certain capacity that can share a wireless channel, for given performance requirements. Alternatively, the system can verify the actual performance results for ECGs, accelerometers, pulse-oximeters etc in a wireless sensor network.

\section{USE CASES}

In this section we present test cases that illustrate the potential performance problems with contention-based access and the need for admission control as well as continuing performance monitoring and control. The first case (Section V.A) illustrates the non-trivial performance problems associated with contention-based access (CSMA). The second case (Section V.B) shows how admission control works in real-time. The length of the test period is also discussed. In the third case (Section V.C), the implemented system is used as an off-line configuration tool to determine how changes of the traffic pattern influence the packet loss ratio. Finally, the alternative to refer a requesting sensor node to a new channel is mentioned in Section V.D.

The testbed in this work consists of TmoteSky sensor nodes running TinyOS 2.1 .0 programmed in nesC [14]. The radio (CC2420) and link layer are compliant with IEEE 802.15.4 LR-WPAN in contention-based access mode. The sensor nodes transmit samples from ECGs, pulse-oximeters and accelerometers with sampling rates from $100 \mathrm{~Hz}$ to $250 \mathrm{~Hz}$. 


\section{A. Performance Problems in Contention-Based Access}

Contention-based access is a challenge for applications that require good and predictable performance. Fig. 5 illustrates what can happen when several sensors access a wireless channel. Three sensors (A, B and $\mathrm{C}$ ) are connected to a coordinator sharing the same channel. The sensors are sampled during a second and the packets are sent back-to-back once a second. The bit rate is $9.6 \mathrm{kbps}$ for each sensor node. Fig. 5 shows the loss ratio during a measurement period for sensor A. During the first part (0-70 seconds) only sensor A is active. The loss ratio is almost zero. Between 70-140 seconds sensor $\mathrm{B}$ also accesses the channel. The average loss ratio experience by sensor $\mathrm{A}$ is 0.03 . During the remaining measurement period all three sensors are transmitting on the same channel. The average loss ratio suddenly rises to 0.40 . Next time the measurement is repeated the loss ratio might be considerably lower.

For a loss-sensitive application, the performance is unacceptable after sensor B, and especially after sensor $\mathrm{C}$, have accessed the channel. The performance degradation may be avoided if the coordinator applies admission control and also maintains performance monitoring and control to protect the quality of service requirements for the existing nodes.

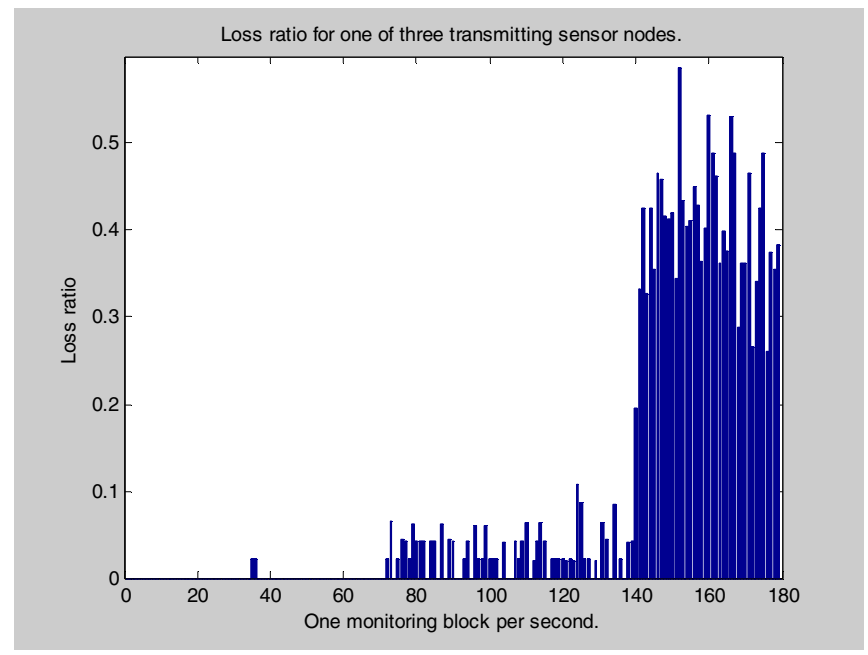

Figure 5. The loss ratio (y-axis) for sensor A node during the measurement period ( $\mathrm{x}$-axis in seconds). At approximately $\mathrm{t}=70 \mathrm{~s}$ sensor $\mathrm{B}$ joins the channel. At $\mathrm{t}=140 \mathrm{~s}$ a third node, sensor $\mathrm{C}$, accesses the channel.

\section{B. Admission Control}

In this test case the coordinator applies admission control when three sensors (accelerometers), one by one, request to join the wireless network (Fig. 6). The sampling rate for the three-axis accelerometer is $200 \mathrm{~Hz}$ per axis and the resulting average bit rate is $9.6 \mathrm{kbps}$. The upper limit for packet loss for each node is set to 0.02 per monitoring block (the block size is around 1 second). The admission test period is 30 monitoring blocks (30 seconds). The measurement sequence is outlined in Fig. 7.

Sensor A requests admission and begins transmitting probe packets. The loss ratio during this test period is zero. Sensor A's request is accepted and it starts transferring data. The loss ratio for the data traffic from sensor $\mathrm{A}$ is almost zero for the period before sensor B requests to join the channel. Table 1 summarizes the loss ratio for each sensor nodes during all test periods and data transfer periods. Losses that exceed the threshold (0.02) are indicated in bold text. It turns out that sensor A and B are accepted, while sensor C is rejected. For a sensor to be rejected it is sufficient that the loss ratio for one of the sensors, including the requesting node itself, exceeds the threshold.

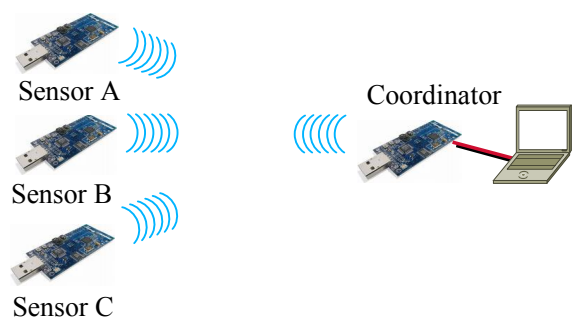

Figure 6. Three sensor nodes connected to the coordinator sharing the same channel.

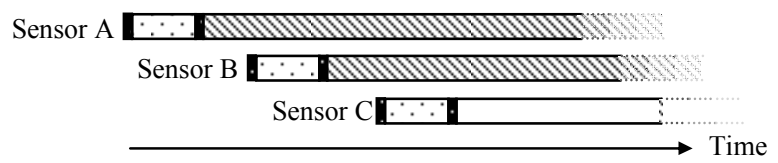

Signaling $\because$ Test traffic $\$$ Sensor data

Figure 7. The measurement sequence for the nodes in Fig. 6.

TABLE I. LOSS RATIO FOR TEST PERIODS AND DATA TRANSFER PERIODS FOR SENSOR A, B AND C.

\begin{tabular}{|c|c|c|c|}
\hline $\begin{array}{c}\text { Test period } \\
\text { sensor A }\end{array}$ & Sensor A & Sensor B & Sensor C \\
\hline $\begin{array}{c}\text { Data } \\
\text { transfer }\end{array}$ & 0.0000 & -- & -- \\
\hline $\begin{array}{c}\text { Test period } \\
\text { sensor B }\end{array}$ & 0.0012 & 0.0085 & -- \\
\hline $\begin{array}{c}\text { Data } \\
\text { transfer }\end{array}$ & 0.0019 & 0.0088 & -- \\
\hline $\begin{array}{c}\text { Test period } \\
\text { sensor C }\end{array}$ & 0.0046 & $\mathbf{0 . 0 4 7 0}$ & $\mathbf{0 . 0 2 5 0}$ \\
\hline $\begin{array}{c}\text { Data } \\
\text { transfer }\end{array}$ & 0.0051 & 0.0083 & -- \\
\hline
\end{tabular}

The length of the test period is a trade-off between, on the one hand, to minimize the disturbance of existing traffic and reducing the response time for the admission verdict, and on the other hand, to receive sufficient performance data. The drawback of a predetermined fixed length test period is that ongoing traffic may suffer from severe performance deterioration. Fig. 8 shows the impact of test traffic on a sensor node during a 30 second test period. The average loss ratio is almost 0.05 , with several peaks around 0.10 , which is unreasonable performance deterioration for an existing node during a test period. To avoid this we use an algorithm that calculates the cumulative moving average of the loss ratio for each incoming performance update i.e., for each monitor- 
ing packet. The test period is interrupted if the cumulative average exceeds a threshold. The cumulative moving average is defined as $\mathrm{CA}_{i}=\left(\mathrm{L}_{1}+\mathrm{L}_{2}+\mathrm{L}_{3}+\ldots+\mathrm{L}_{i}\right) / i$, where $\mathrm{L}_{i}$ is the loss ratio for monitoring block $i$. The algorithm is applied to three examples of test periods in Fig. 8-10. The cumulative averages for the first five blocks in Fig. 8 are $\mathrm{CA}_{1}=0.059$, $\mathrm{CA}_{2}=0.035, \mathrm{CA}_{3}=0.032, \mathrm{CA}_{4}=0.042$ and $\mathrm{CA}_{5}=0.042$.

If the rule for admittance is to allow maximum three consecutive updates of the loss ratio above the threshold $(0.02)$, the test period will be interrupted after the third block. An additional requirement that the loss ratio for a single block may not exceed 0.05 would in this example mean that the test period is stopped after the first monitoring block.

A slightly different loss pattern is depicted in Fig. 9 (sensor C's loss ratio during a test period). The cumulative average for the first seven blocks are $\mathrm{CA}_{1}=0.0118, \mathrm{CA}_{2}=0.0119$, $\mathrm{CA}_{3}=0.0159, \mathrm{CA}_{4}=0.0240$ and $\mathrm{CA}_{5}=0.0216, \mathrm{CA}_{6}=0.0201$ and $\mathrm{CA}_{7}=0.0206$. In this case, the test period terminates after the $6^{\text {th }}$ monitoring block and the request is rejected.

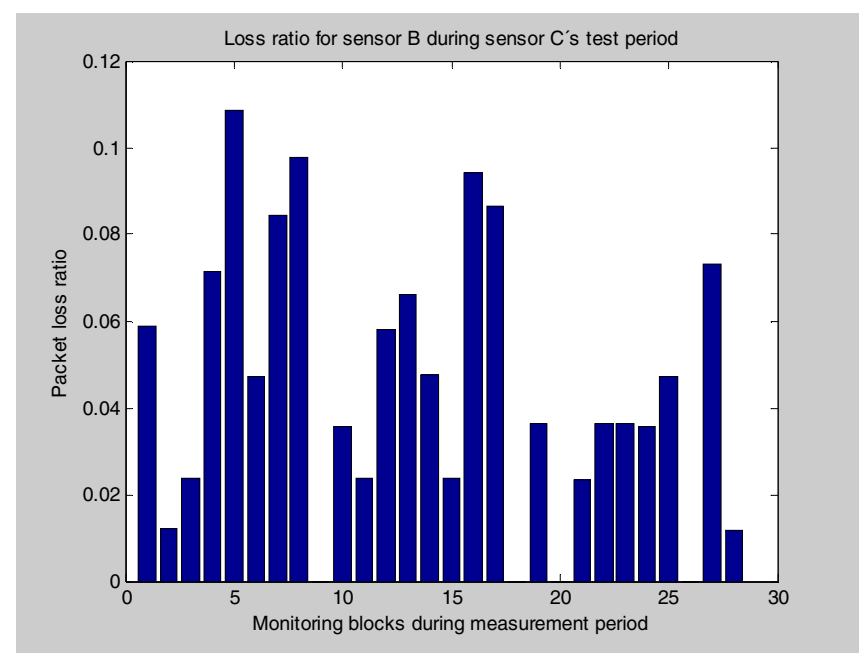

Figure 8. Loss ratio per monitoring block experienced by sensor B during the third sensor's (sensor C) test period. The average loss ratio is 0.047 .

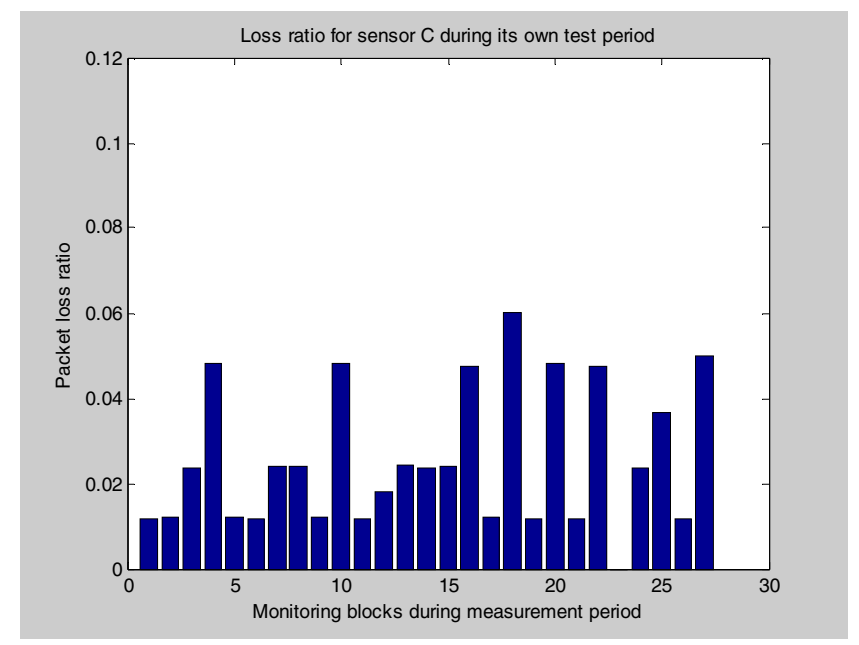

Figure 9. Loss ratio per monitoring block experienced by sensor $\mathrm{C}$ during its own test period. The average loss ratio is 0.025 .

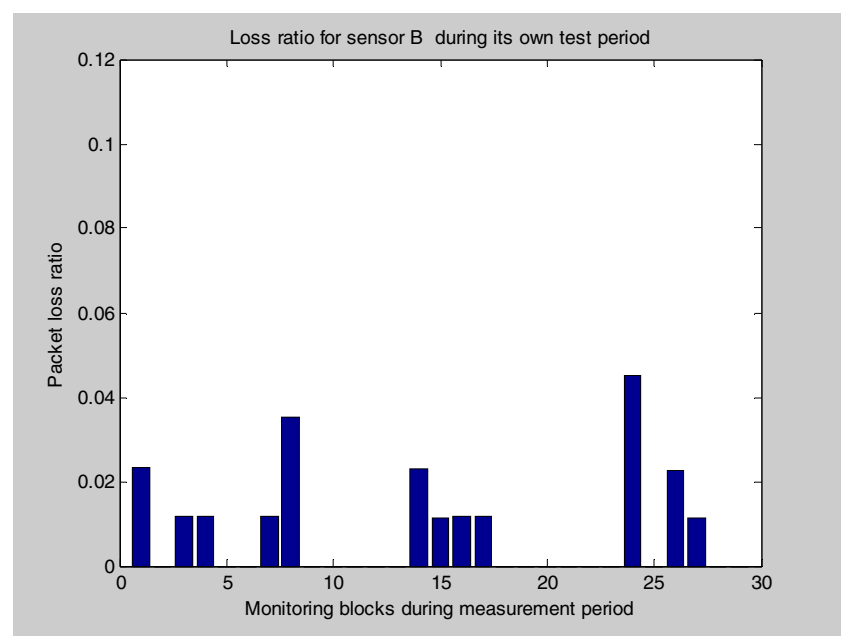

Figure 10. Loss ratio per monitoring block experienced by sensor B during its own test period.

\section{Traffic Patterns and Channel Access}

Packet loss in contention-based wireless networks is sensitive to the traffic pattern from the individual sources. Assume that two nodes collect samples and transmit the samples as a train of packets periodically once a second. If the nodes transmit the packet trains without overlap in time, the risk for losses due to collisions is low. However, the loss probability will increase if the packet trains happen to coincide. The dynamics of the traffic patterns in a network may from time to time lead to losses that exceed the accepted level after the admission test periods. The unpredictability of performance deterioration in wireless contention-based networks means that admission control must be combined with continuous traffic monitoring and control to be able to maintain the desired performance goals.

We have performed tests to study the impact of changes in traffic pattern on packet loss. Sensor node A collects and stores samples during a second. The samples are encapsulated in packets and transmitted back-to-back. The total time to transmit the packet train depends on the sampling rate, the sample size and the packet size. In this case the sensor node sends a packet train of 43 packets with a packet size of 28 bytes, which corresponds to a throughput of $9,6 \mathrm{~kb} / \mathrm{s}$. The total time to send the packet train was around $500 \mathrm{~ms}$. A second node, sensor B, starts transmitting probe packets. It sends a train of packets once a second during the test period. The starting time for each train is shifted $50 \mathrm{~ms}$ after ten seconds. This is repeated ten times, which means that the total time shift of the packet trains is around $500 \mathrm{~ms}$. The basic idea is to let the packet trains from sensor B slide over the packet trains from sensor A. Fig. 11 illustrates this convolution-like procedure.

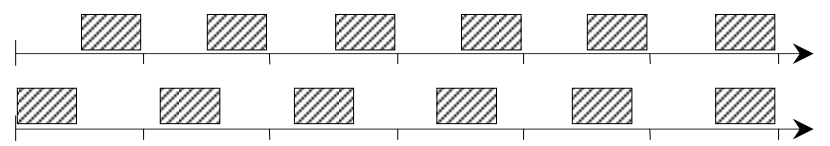

Figure 11. Sensor A (the upper part) sends packet trains periodically every second. The starting times of the trains transmitted by sensor B (lower part) are shifted in time so that they slide over the packet trains from sensor A. 
Fig. 12 shows the loss ratio for sensor B. After 10 monitoring blocks (10 seconds) the starting time is shifted $50 \mathrm{~ms}$. The average loss ratio for the first half of the measurement period is below 0.01. It rises to 0.10 for block $81-90$ and 0.17 for block 91-100. The highest losses occur when the packet trains from the two sensors coincide in time. This convolution-like test might be inappropriate to use in an operating network but is useful for out-of-service configuration and dimensioning tests to estimate a worst case loss ratio.

The traffic pattern for a channel e.g., the starting times of packet trains, is a stochastic process that may result in random losses from zero up to $25 \%$ in this case. Due to the unpredictability of contention-based wireless access continuous performance monitoring and control is needed to maintain the desired performance levels.

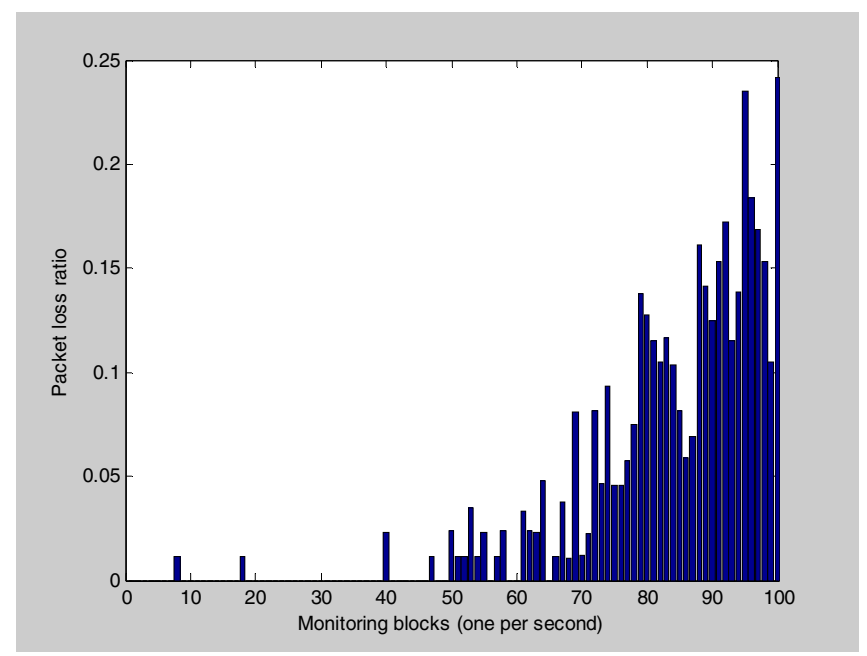

Figure 12. Loss ratio for sensor B. The peak values occur when the packet trains from sensor B coincide in time with the packet trains from sensor A.

\section{Redirecting to Another Channel}

When a sensor node's request to join the network is rejected there are two alternatives. The node may back off for a while and try once again later. Alternatively, the coordinator may refer the sensor to another radio channel. This feature has been successfully implemented and tested.

\section{CONCLUSIONS}

Wireless sensor networks have today emerged as a feasible infrastructure for healthcare applications. This paper has addressed the non-trivial performance problems related to contention-based access wireless channels. We have presented a method for admission control, based on transmission of probe packets during a test period, as a component of a performance management system. This system can be used as a tool for dimensioning and configuration, as well as realtime admission control and continuous performance management of wireless sensor networks.

The length of the test period is a trade-off between minimizing the disturbances on existing traffic, and receiving sufficient performance data for the admission verdict. The proposed algorithm uses a cumulative moving average of the loss ratio for the traffic from each sensor node to decide whether to reject an admission request and interrupt the test traffic, or to allow the sensor use the network. The test results show that admission control can improve the level and predictability of the performance of wireless sensor nodes. A final conclusion is that continuous performance monitoring and control is needed to maintain the desired performance levels.

\section{REFERENCES}

[1] "Zigbee wireless sensor applications for health, wellness and fitness", Zigbee Alliance, March 2009.

[2] R. Carroll, R. Cnossen, M. Schnell, and D. Simons, "Continua: an Interoperable Personal Healthcare Ecosystem", IEEE Pervasive Computing, Vol.6, No 4, October-December 2007.

[3] A. Brandt (Zensys Inc) and G. Porcu (Telecom Italia), "Home Automation Routing Requirements in Low Power and Lossy Networks", Internet Draft, September 2009.

[4] I. Más and G. Karlsson, "Probe-based admission control for differentiated-services internet", Computer Networks 51, pp.39023918, 2007.

[5] P. Ramanathan, D. Moore, and C. Dovrolis "What Do Packet Dispersion Techniques Measure", In Proceedings of IEEE INFOCOM, 2001, pp. 905-914, April 2001, Anchorage, Alaska, USA.

[6] M. Jain and C. Dovrolis, "Pathload: a measurement tool for end-toend available bandwidth", Passive and Active Measurements Workshop, March 2002, Fort Collins, USA.

[7] S. Ekelin, M. Nilsson, E. Hartikainen, A. Johnsson, J-E Mångs, B. Melander, and M. Björkman, "Real-Time Measurement of End-toEnd Available Bandwidth using Kalman Filtering", IEEE NOMS 2006, Vancouver, Canada.

[8] T. Sun, L. Chen, G. Yang, M. Y. Sanadidi, and M. Gerla, "SenProbe: Path Capacity Estimation in Wireless Sensor Networks" SenMetrics 2005, July 2005, San Diego, USA.

[9] D. Gupta, D. Wu, P. Mohapatra and C-N. Chuah, "Experimental Comparison of Bandwidth Estimation Tools for Wireless Mesh Networks", IEEE INFOCOM Mini-Conference, April 2009.

[10] Y. Yang and R Kravets, "Contention-Aware Admission Control for Ad Hoc Networks" Mobile Computing, IEEE Transactions on Volume 4, Issue 4, July-Aug. 2005.

[11] Ian D. Chakeres and Elizabeth M. Belding-Royer, "PAC: Perceptive Admission Control for Mobile Wireless Networks", International Conference on Quality of Service in Heterogeneous Wired/Wireless Networks (QShine), October 2004, Dallas, USA.

[12] I. Orhan, A. Gonga, and T. Lindh, "An End-to-End Performance Meter for Applications in Wireless Body Sensor Networks", International Workshop on Wearable and Implantable Body Sensor Networks, June 2008, Hong Kong.

[13] T. Lindh and I. Orhan, "Performance Monitoring and Control in Contention-Based Wireless Sensor Networks", International Symposium on Wireless Communication Systems, September 2009, Siena, Italy.

[14] D. Gay, P. Lewis, R. von Behren, M. Welsh, E. Brewer, and D. Culler, "The nesC language: A holistic approach to networked embedded systems", Proceedings of the ACM SIGPLAN 2003 conference on Programming language design and implementation, San Diego, USA. 